uniform motion of a rotating baroclinic fluid, such as the atmosphere, is unstable in almost all circumstances, even when convectively stable. Previous attacks on the problem of dynamical instability when there are no velocity discontinuities (velocity discontinuity always gives rise to instability, at least on a small scale) have lacked generality. They discussed essentially 'overturning' in a vertical plane and obtained the well-known criterion, negative absolute vorticity in isentropic surfaces. But another type of disturbance exists, corresponding to 'overturning' in a plane sloping upwards toward the pole at an angle less than that of the isentropic surfaces. Long-wave disturbances are almost always unstable. In each case there exists one particular disturbance which grows faster than any other and becomes apparent on synoptic charts by a process analogous to 'natural selection'. The long waves observed are the form of breakdown, by this process, of the whole troposphere. Smaller secondary disturbances are the form of breakdown, by a similar process, of baroclinic cloud sheets, that is, 'frontal' regions. 'There is good numerical agreement between theory and observation regarding wave-length, wave-velocity, growth-rate, and structure of these disturbances. Dr. Eady concluded by discussing briefly some of the difficulties encountered in attempting to extend the theory to make possible the computation of weather changes (more precisely, of most probable changes), and also mentioned one way in which orography causes irregularity in the mean flow at all levels.

In the absence of Mir. C. H. B. Priestley, Dr. A. G. Forsdyke gave an account of his paper, "Heat Transport and Zonal Stress Between Latitudes" (Quart. J. Roy. Met. Soc., 75, 28; Jan. 1949). Mir. Priestley was concerned with estimating, from empirical evidence given by radio-sonde winds and temperatures, the actual poleward transport of heat and momentum referred to above. The flux of heat can conveniently be divided into (i) a component associated with the mean flow; (ii) a 'toroidal' (meridional) component associated with net poleward flow at low levels, equatorward flow at high levels; (iii) an eddy-flux associated with transient disturbances. Since for this purpose heat may be regarded as a conservative quantity, the eddy-flux of heat is proportional to the correlation between poleward motion and excess heat content. It is convenient to divide the latter into sensible heat (measured by temperature), and latent heat of water vapour. It would be desirable if this eddy-transport could be evaluated at a large number of stations, and it was one of Mir. Priestley's objects to set an example by computing it for one station, namely, Larkhill, England. He found that transport of heat in latent form is about the same as that in sensible form. He also noted a curious negative transport, that is, towards the equator, in the upper troposphere, while in the stratosphere the transport is towards the poles. He also computed the toroidal flux, but there appears to be some doubt (expressed by Mr. J. S. Sawyer and Dr. Eady) regarding the validity of his formula.

Momentum (or angular momentum) is not a conservative quantity for individual particles. Nevertheless, the total transport of momentum across a circle of latitude over all heights is given by Jeffreys's formula, which is, in fact, simply the total Reynolds's stress. Mr. Priestley evaluated separately components which can be regarded (formally) as eddyflux and toroidal flux. The latter is considerably the smaller. He found that the total flux is in the right direction in winter, but much smaller and often negative in summer. On the whole, it is of about the right order of magnitude.

Mr. Priestley has not evaluated the transport associated with the mean flow. Evaluation at one station would be of little value, since on integration round a circle of latitude the contributions would largely cancel.

Dr. R. C. Sutcliffe gave an illustrated account of a preliminary attempt to sort out types of largescale flow empirically. $\mathrm{He}$ suggested that the atmosphere can 'solve its own equations' in a variety of ways, yielding types of flow pattern which persist for considerable periods ; from time to time it 'flips over' relatively quickly from one basic pattern to another. $\mathrm{He}$ did not indicate, however, why this takes place, except to suggest that it is associated with seasonal changes.

Sir David Brunt emphasized the importance of ealculations such as those of Mr. Priestley. $\mathrm{He}$ referred to several early calculations, including those of Mir. E. Gold, indicating radiative cooling of the upper troposphere. He suggested that some meteorological problems cannot yet be solved because of ignorance regarding the correct boundary conditions. Mir. J. S. Sawyer emphasized the importance of travelling disturbances in determining the general circulation. Prof. P. A. Sheppard, referring to strong winds (and hence appreciable energy in spite of low density) in the upper stratosphere, indicated the possibility of events at these levels affecting the circulation below in the long run.

Mir. A. W. Brewer said that accurate humidity measurements, with the Dobson-Brewer frost-point hygrometer, suggest, on account of the extreme dryness of the stratosphere, that the troposphere and stratosphere are two different worlds. Nieasurements of helium content show no appreciable variation up to at least $22 \mathrm{~km}$., so that there must be some diffusion across the tropopause; but it cannot be large or the very steep gradient of vapour content would be destroyed. He suggested that stratospheric air in middle latitudes originates above the tropopause in low latitudes, where even saturated air has very little vapour content. He indicated some of the difficulties involved in this theory, since it requires rather rapid radiative cooling in the air subsiding along the tropopause ; further, if purely zonal flow is postulated, angular momentum should be conserved (contrary to observation) during transit.

In his concluding remarks Dr. Eady referred to the changes in the tropospheric motion to be expected if the mean stratospheric temperature changes, with consequent alteration in the mean height of the tropopause.

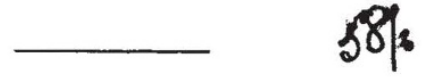

\section{HETEROGENEOUS CATALYSIS}

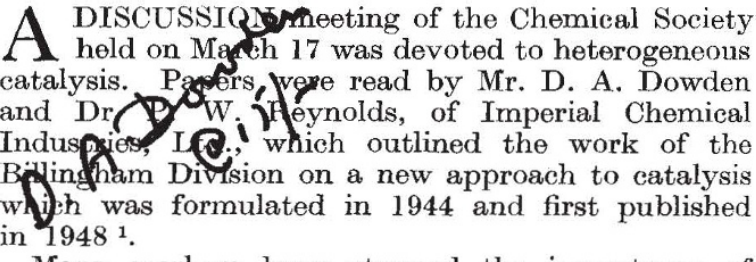

Many workers have stressed the importance of relationships between the dimensions of reacting molecules and the crystal parameters of the catalysts 
which are active for their conversion. The point has been emphasized by the careful work of Beeck and his co-workers ${ }^{2}$ on evaporated Group VIII metal films in ethylene hydrogenation. They found a fivefold greater activity for oriented nickel films than for random films, and they ascribed this difference to preferred spacings exposed in the oriented films. Following this indication, Beeck ${ }^{3}$ related the activity of metal hydrogenation catalysts to their crystal parameters and demonstrated the predicted high activity of rhodium.

It seems certain that such spatial considerations must play their part; but even if the suggested geometrical relationships are necessary for high activity, they are certainly not sufficient. Copper, silver and gold, for example, have crystal parameters which would suggest a high activity, whereas, in fact, there is little or none. Moreover, it is always possible that these geometric correspondences are only incidental, and the prevalent tendency to hunt among the Miller indices for a plane with a promising spacing is viewed with suspicion. It has been the aim of the Billingham work to establish alternative correlations between the catalytic activity and physical structure of solids which may be more fundamental than these.

In the simplest example of hydrogenation over metallic catalysts, the activity of the Group VIII metals is attributed to the unfilled electron-levels in their $3 d$-, $4 d$ - and $5 d$-bands. The relative inactivity of copper, silver and gold is thus explicable. It becomes of special interest to study the alloys of the Group VIII metals with these Group I $b$ metals and with one another. The principal effort is being directed to the study of spectroscopically pure metallic foils ; but results already obtained on industrial catalysts in the nickel-copper system give strong support to the theoretical picture. The system exhibits a continuous series of solid solutions; the lattice parameter-composition relationship does not depart appreciably from Végard's law. Beeck's correlation would suggest a steady increase in the activity of the nickel by the expansion of its lattice through the inclusion of copper. The electronic picture, on the other hand, would suggest a marked fall in the activity of nickel as the valency electrons of the Group I $b$ metal fill up the holes in its $3 d$-band. In pure alloys at equilibrium, these holes are just filled up by 60 atomic per cent of copper. The ferromagnetic properties, as measured by the large magnetic susceptibility at a fixed field strength, disappear at the same composition. So also should the catalytic activity.

Alloy-skeletal catalysts, derived from coppernickel-aluminium alloys by extraction with caustic soda, and reduced copper-nickel oxide on kieselguhr catalysts, have been examined in benzene and styrene hydrogenation. In both series there was a parallel decline in activity and magnetic susceptibility as the copper : nickel ratio increased, the values becoming very small at 30-40 atomic per cent of copper. The occurrence of the critical composition at a lower copper content than would have been expected from the properties of the pure alloys is undoubtedly due to the complexity of the catalysts in question; but these results do show that the catalytic activity and the number of holes in the $d$-band fall away together, and that they do this while the crystal parameter is increasing slightly in the direction of increased activity on the simple geometric view.

Catalysis must be preceded by chemisorption of the reactant, so that the catalyst crystallites may be considered as sources or sinks for the electrons transferred or exchanged in the ionic or covalent bonding which is involved. The electron band theory of solids, the classical ionic lattice theory and the rapidly expanding knowledge of defect structures enable these processes to be viewed with a new precision.

Provided that surface and volume diffusion steps are very fast, the rate-controlling step in a catalysed reaction must be either the chemisorption of a reactant, a reaction in the sorbed state, or the desorption of a product. The form of the free-energy curves representing these steps ${ }^{4}$ is such that the free energy of activation is always lowered, other things being equal, by bringing the initial and final states close together in space, by reducing the free energy of the initial state, or by increasing that of the final state. An active centre would then appear as a region of special geometry which makes possible this proximity of initial and final states, and the free energy would correspond to the strength of the bonds with the surface. This bonding may be homopolar or heteropolar, but in either case appropriate levels of minimum energy must be found for the electrons exchanged or transferred at the interface when the atoms, radicals or ions are adsorbed.

Modern theory provides a detailed knowledge of these processes. Excluding surface states from consideration, the donation of an electron to the catalyst crystallite by an adsorbate, whatever the mechanism, must raise the energy of the Fermi surface and is equivalent to the addition of an electron to the lowest empty level. This process is easiest when there is only a small difference between the ionization potential of the particles at the surface $\left(I^{\prime}\right)$ and the metal work function $(\varphi)$, and when the density of levels at the Fermi surface is large. If the sorbate at the surface is considered to be a surface phase, the electrons of which are in equilibrium with those of the metal through a dilute electron gas, then the ratio of ionized to neutral species in the surface phase $\left(C_{A}+/ C_{A}\right)$ is proportional to

$$
\exp -\left(I^{\prime}+\mu_{e}^{M}\right) / K T \text {, where } I^{\prime}=I-\Delta U^{+},
$$
and $\mu_{e} M=-\varphi-\frac{1}{6} \pi^{2} K^{2} T^{2}\left(d \log _{\theta} g(\varepsilon) / d \varepsilon\right)_{\varepsilon}=-\varphi$,

where $\mu_{e} M$ is the thermodynamic potential per electron per unit volume, $I$ is the ionization potential of the neutral particle in vacuo, $-\Delta U^{+}$is the adsorption energy of the cation, $g(\varepsilon)$ is the density of electron levels per unit volume, and $\varepsilon$ is the metal electron energy. In a given system $\left|\Delta U^{+}\right|$is a maximum (and $I^{\prime}$ a minimum) when the ion has acquired the greatest number of lattice atom nearest neighbours, so that the active centre is a position where the ion can be located interstitially at the surface, or occupy, as closely as possible, a vacant lattice point at the surface. The Helmholtz free-energy of activation for ionization at such a centre is given by :

$$
\Delta F^{*} \propto I+\mu_{\theta} M-\text { constant } \times \Delta U^{+},
$$

and the factors which contribute to a large surface concentration of cations also increase the rate of cation formation.

If it be assumed for simplicity that the forces contributing to $\Delta U^{+}$are Coulombic (image) forces and practically independent of the metal, then positive ion formation is most favoured and fastest on metals and alloys exhibiting a large electron exit work function and a large positive value of ( $d \log _{6}$ $g(\varepsilon) / d \varepsilon)$ at the Fermi surface. It will be favoured also by crystallization in a manner which exposes 
the planes of least dense packing, or more densely packed planes with a high concentration of vacant sites, and by the addition of traces of electronegative substances. The data available suggest that the requisite metallic properties are to be found in metals and alloys having holes at the top of the $d$ - or $f$-bands. (When both the cation radius and $I$ are large, as with some saturated molecules, ionization may more easily occur indirectly by electron transfer with some more easily formed cation, for example, $\mathrm{H}^{+}$.)

By similar reasoning it is demonstrable that negative ion formation requires the same geometry for the active centre, but a small electron exit work function and a large negative value of $\left(d \log _{e} g(\varepsilon) / d \varepsilon\right)$ at the Fermi surface. It will be promoted by traces of electropositive additions.

The concentration and rate of formation of a covalently bonded species depend similarly on the bond strength. Pauling's older concepts ${ }^{5}$ show that the bond strength decreases as the $d$-orbital content of the hybridized orbitals diminishes, whereas according to his later scheme $e^{6}$ the number of empty 'atomic' orbitals drops to zero as the $d$-band is filled. The number of unused surface 'bonding' orbitals continues to fall as more $s$-electrons are added to the metal. Since these orbitals are required for occupation by electrons in the process of chemisorption, a rapid fall in activity occurs at the alloy composition where the $d$-band is filled, followed by a much slower decline with further increase in the electron : atom ratio. The band theory of metals leads to the same conclusion, since the number of canonical forms of low excitation energy of the chemisorbed complex must increase with increasing electron-level density, leading to enhanced resonance energy and greater bond strength. As entropy can be put proportional to the logarithm of level density, it is suggested that the entropy of activation decreases as bands of high level density are filled. Real chemisorptive bonds involve Coulomb forces, exchange forces and Van der Waals forces, and it is convenient that when the mixed bond involves a tendency to positive ion formation, all three vary in the same way with the properties of the $d$-band holes.

These relationships between the electronic structure of the solid and the rate and energy of formation of chemisorbed species can now be applied to the prediction and explanation of catalytic activity in reactions controlled by the rate of formation of ions or radicals or covalently bonded complexes, or wherein the rate-controlling step depends on the concentration of such species on the surface. By this means catalytic activity can be directly related to the energy and density of levels at the Fermi surface. The correlation explains the activity trends in binary and multicomponent alloys, where the rate of fall in activity with added $s$-, $p$-metals increases with the valency of the latter; it provides an interpretation of the changes in activity at the phase boundaries in Hume-Rothery alloys; and it goes a long way to explain the much-disputed magneto-catalytic effects of Hedvall and the observed effects of order-disorder transformation in alloys. Catalyst poisoning is also elucidated; it is now clear that the poison may be effective not only by obscuring the surface, but also through its effect on the electron work function of the solid and on the unfilled electron levels and orbitals within it.

Extension of the same principles to catalysts which are semi-conductors or insulators is more difficult, but considerable progress has been made. The same considerations show that positive ions are most easily formed on defect conductors or on insulators with easily reducible cations, particularly when the sub. stance contains a stable component with cation defects. Conversely, negative ions are most easily formed on excess semi-conductors or on insulators with easily oxidized cations or where stable anion defects are present. (It is possible that excess semiconductors under high hydrogen pressures function as metal catalysts.) Insulators-which neither accept nor donate electrons easily-function by chemisorbing reactants as positive and negative ions; proton exchange mechanisms are prominent, and these proceed most readily at cation defects.

In discussion, Dr. D. D. Eley (Bristol) presented experimental data on the ortho-para hydrogen conversion at the surface of palladium-gold alloys, which clearly indicated a rapid drop in activity at about 50 atomic per cent of added gold, that is, at the composition corresponding to the complete filling of the $d$-band in palladium. The drop in activity is accompanied by a large rise in activation energy and a decrease in the frequency factor, to be contrasted with the more common parallel changes in these variables. The fact that hydrogen poisons the reaction on pure palladium would appear to indicate that hydrogen itself can provide electrons to fill up the $d$-orbital of the palladium.

Dr. E. B. Maxted (Bristol) discussed evidence which showed that poisoning of platinum catalysts by transitional metal ions occurs only when the added ion has all five orbitals of a $d$-shell immediately preceding $s$ - or $p$-valency shells electronically occupied. Regularities also exist in the correlation between the catalytic activities and magnetic susceptibilities of the platinum metals.

Dr. J. S. Anderson (Harwell) presented some data on the electrical properties of semi-conducting zinc oxide which suggested that at very low partial pressures of oxygen the excess zinc atoms may aggregate into small regions of two-dimensional metal.

${ }^{1}$ Dowden, Research, 1, 239 (1948).

${ }^{2}$ Beeck, Smith and Wheeler, Proc. Roy. Soc., A, 177, 62 (1940).

${ }^{3}$ Beeck, Rev. Mod. Phys., 17, 61 (1945).

${ }^{4}$ Lennard-Jones, Trans. Farad. Soc., 28, 333 (1932).

"Pauling, "The Nature of the Chemical Bond"'(1942).

'Pauling, Rev. Mod. Phys., 20, 112 (1948).

\section{COLONIAL GEOLOGICAL SURVEY WORK}

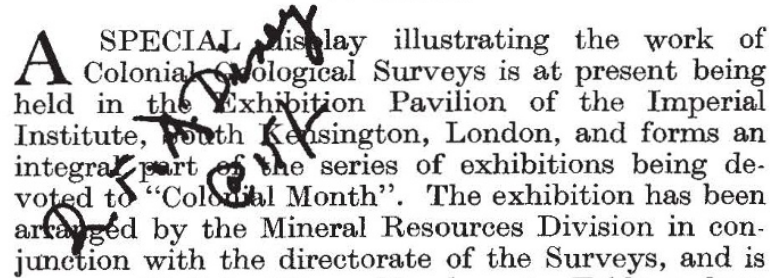
open until July 20 on Mondays to Fridays from 10 a.m. to 4.30 p.m., and on Saturdays from 10 a.m. to 1 p.m.

Since January 1, 1947, all official matters relating to the development of Colonial geological survey work have been the responsibility of Dr. F. Dixey, director of Colonial Geological Surveys and geological adviser to the Secretary of State for the 